$\begin{array}{cccc}\text { S sciendo } & \text { International Conference KNOWLEDGE-BASED ORGANIZATION } \\ \text { Vol. XXV } & \text { No } 1 & 2019\end{array}$

\title{
THE APPLICABILITY OF THE FIRST STAGE OF 5S METHODOLOGY TO IMPROVETHE QUALITY OF PROCESSES IN MILITARY ORGANIZATIONS
}

\author{
Nicolae MORO, Florin ILIE \\ "Nicolae Bălcescu" Land Forces Academy, Sibiu, Romania \\ nicu.moro@yahoo.com, ilieflorinv@yahoo.com
}

\begin{abstract}
The paper addresses the first step of the 5S methodology, a widespread methodology in organizations that has as its primary focus the continuous improvement of the quality of material goods production processes or customer service delivery. This methodology has a very good application area within the defence system because this field is essentially based on a very strong organizational culture with a number of specific rigor and standards that contribute to the implementation of the five concrete stages of this methodology. In a synthetic analysis of these types of organizations, it is obvious that these activities necessary for the application of this useful tool for the organization of jobs have a solid foundation but it should be restructured according to the criteria set out in the 5S methodology.
\end{abstract}

Keywords: quality, kaizen, sort, red tag.

\section{Introduction}

The concept of continuous improvement through the Kaizen management was born and gradually developed in Japan between 1950 and 1980, but the name was officially recognized and registered as a US brand in 1985 when professor Masaaki Imai, the founder of the Kaizen Institute, published the book "Kaizen - the key to Japan's competitive success" disseminating the experience gained for several decades of practical application of these principles to mankind. Even for a part of Japanese managers, the Kaizen theoretical concept was just a simple noun used in Japanese, expressing a conception that urged to do anything "always better".

Professor Masaaki Imai's works and experiences in this area made him gain the world reputation of "Guru Kaizen" or "the father of continuous improvement". The book that defines the philosophy and practices of change in the ever-better, "Kaizen: The key to Japan's competitive success" and that was published at McGraw-Hill Publishing House in 1986, has been translated into more than 20 languages and published in more than 30 countries with an impact especially on the concepts, approaches and behaviours of a large number of companies (organizations) and managers who adopted the Kaizen techniques and principles [1].

A few years later, in 1997, professor Masaaki Imai published his second book on Kaizen, "Gemba Kaizen: A Commonsense, Low-Cost Approach to Management" at the prestigious McGraw-Hill Publishing House, with the same success and recognition as well as his first book. He discusses the cost advantages and efficiency analyses of applying this type of management.

The Kaizen management system, whose success was demonstrated in Japan, is a 
concept initiated by Japanese managers and has the meaning of Kai = change and Zen $=$ for the better, in the sense of a continuous, day-to-day, process improvement. In contrast to the Western concept, which involves a total change at high intervals of large resources, Kaizen seeks a slow, gradual (process-based) improvement, but continuous, with visible effects from process to process.

\section{The structure of the 5S methodology}

One of Kaizen's specific tools for an organization is to organize the workplace and establish and comply with the visual management standards. In order to prove their effectiveness, processes in areas in which visual management is applied must be properly identified, analyzed, controlled and standardized. But to be able to apply this logical and rational approach, there is a need for order and cleanliness in the sector in which this process takes place.
A useful tool for organizing jobs is 5S, which is essentially a structured program to achieve systematically organization, cleanliness and standardization at work, thus contributing to improved productivity, reducing quality and security issues of work, being a method involving employees on all hierarchical levels through the use of standards and discipline. Being a native Japan method for organizing, cleaning, developing and sustaining a productive workplace, the name comes from five Japanese words that begin with the sound S: Seiri, Seiton, Seiso, Seiketsu and Shitsuke.

This technique was immediately taken over by organizations from the powerful Western economies, and by translating the Japanese words into English, an identical designation of the technique was obtained: Sort, Straighten, Shine, Standardize and Sustain.

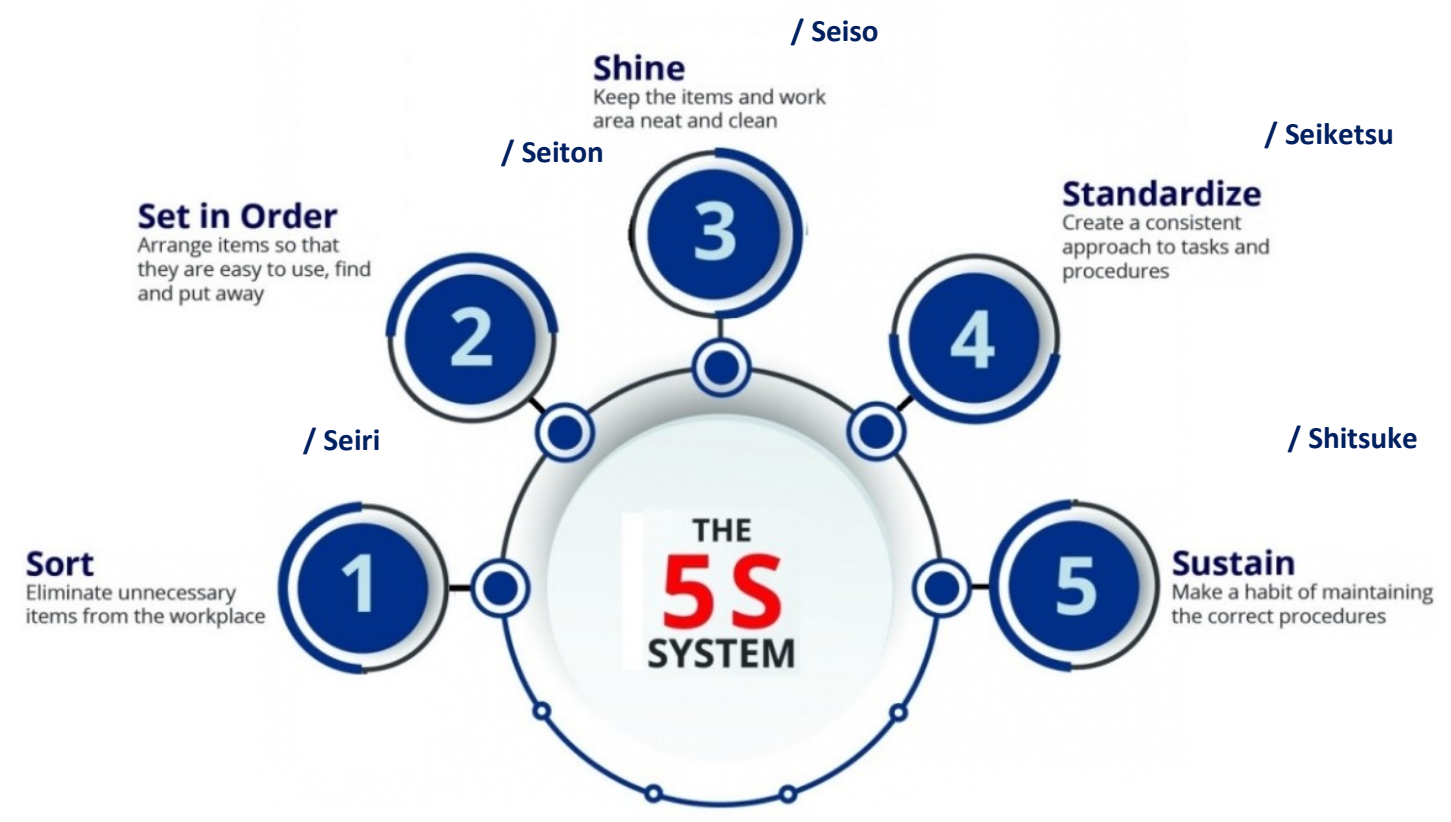

Figure 1The significance of $5 \mathrm{~S}$

If the first three stages of the $5 \mathrm{~S}$ methodology describe Hard approaches that involve tangible elements or assumed organizational and technological aspects of processes, having an impact on the workplace layout and productivity in general, the latter two stages involve the use of Soft methods where the emphasis is on developing skills and habits, team working rules, methods for accepting change, and reducing resistance to change, impacting on the observable behaviours of those involved 
and the acceptance of organizational culture. Overall, the 5S methodology can be synthesized in mathematical form in an

elementary arithmetic calculation operation of the type:
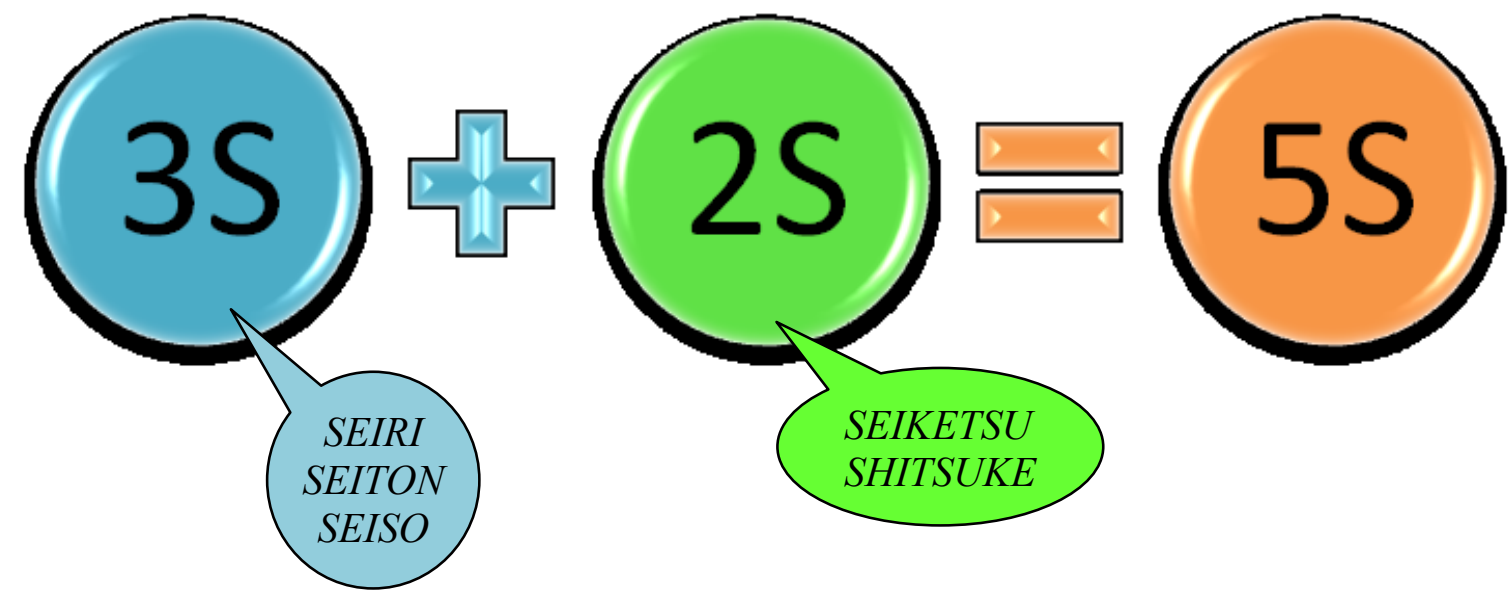

Figure 2 Mathematical formula of the structure of the methodology5S

\section{Stage 1 - Sort and remove unnecessary objects}

The 5S process starts with Seiri (Sort), which is a laborious activity, consuming physical and mental resources but absolutely necessary for the following steps. This first step of the process refers to the release of the work space and the elimination of all unnecessary materials and objects (e.g. obsolete work programs, test pieces, unusable or rarely used documents, old or defective tools, unused accessories, files/books borrowed and not returned to the right owners, etc.). Sorting has an impact on the level of people's mentality in the workplace, because they have to give up the habit of tightening and storing unnecessarily the kind of material and objects having in mind the idea that they will ever need them or for reasons of emotional attachment [2].

All these unnecessary work items on a workstation occupy a lot of space, and besides the fact that purchase costs were required at some point, many of them involve additional administrative costs necessary for their inventory, handling costs, cleaning, storage costs, etc. Even worse is the fact that agglomeration of these objects, reducing the space of movement, can lead to the risk of accidents with consequences for employees and the employer. Unnecessary crowding can also cause problems in finding the right information or objects immediately. Specialty studies have shown that each employee consumes an average of about 1 hour per day for searching and finding what he/she needs while working at job.

Sorting is the right choice in the sense that:

- what is needed must be preserved;

- what is useless should be removed from workplaces.

Sorting and removing items that are no longer necessary and avoiding disorder will have an immediate impact on the state of mind, but will also gain space and sort objects stored for maximum efficiency. This sorting has to be done in all areas and here it is understood on, under, in and after tables/work benches, cabinets, racks, machines, equipment, corners, closets, warehouses, etc.

This type of activity can also be performed in office areas where you can start by checking the furniture and office equipment (old printers, damaged telephones and speakers, used document destroyers, etc.), used supplies (empty printer cartridges, calendars, posters, advertisements, etc.) or personal items (photos, cups, etc.) that are used. 
The sorting and marking of necessary or unnecessary objects can be done using different colour labels, depending on the frequency of use or the approach to managing the organization. Usually the symbolism of colours is the following:

- red labels are used to mark those objects that must be permanently removed from the work area or those objects where there is doubt as to their need, frequency of use or quantity. The rule is that "if you doubt, put a red label".

- green labels are used to mark those necessary materials that are indispensable for the day-to-day work and are available at the workplace. Their marking is necessary in order not to be confused with those objects that must be removed permanently. One element to consider is the optimal amount of such items because too much can cause difficulties in keeping the workplace;

- yellow labels are used to mark those objects that are rarely used but are not to be discarded and which are kept in areas near the workplace (in temporary or quarantine storage areas) and are easily accessible when you need to use them. The average non-use time of these materials is over 48 hours. It is very important that this place be clearly identified and separated and physically disposed in an area that is rarely used or not in the main directions of movement of employees or materials.

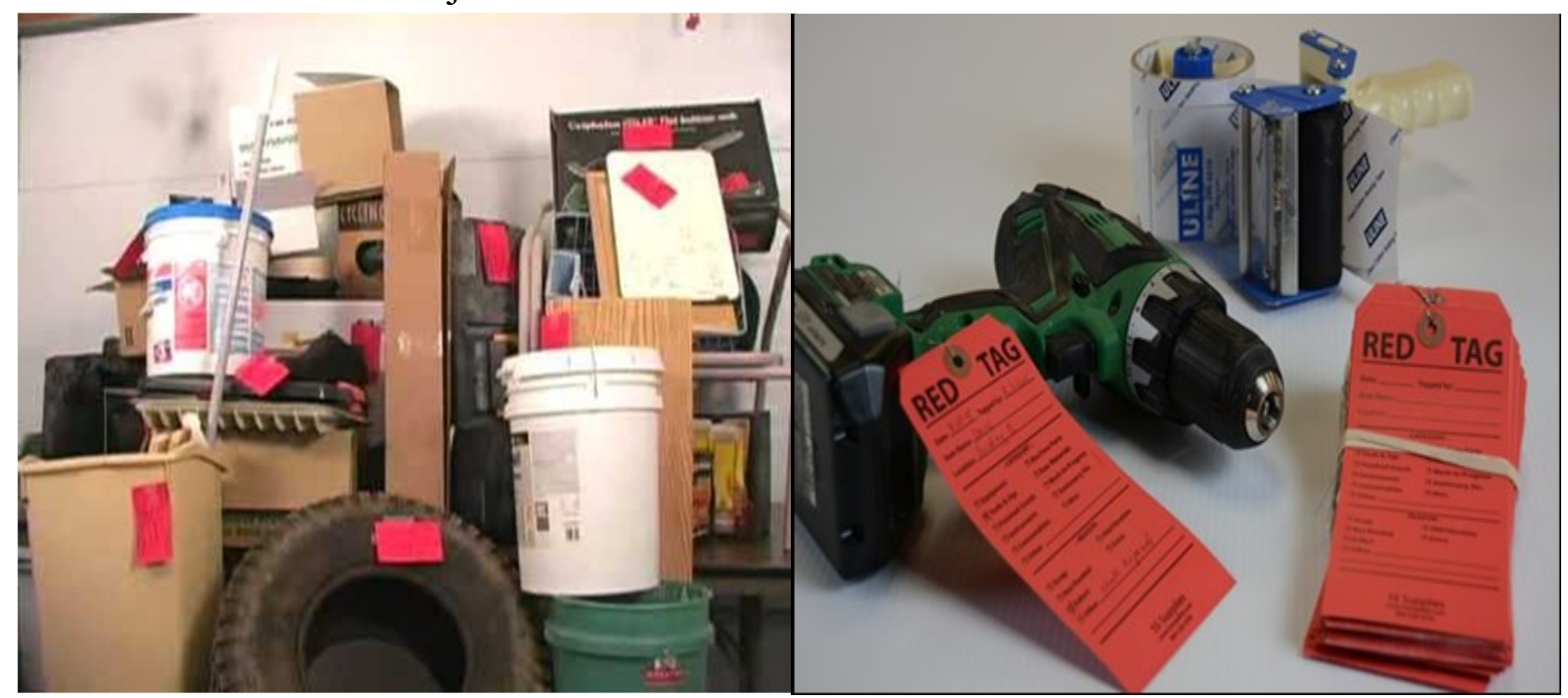

Figure 3 Label unnecessary objects with red labels

Determining which of the three colour labels falls into the workplace objects is the individual task of the employees because they have the best knowledge of the working needs. Each label, it does not matter what colour it is, must include a series of data necessary to identify the name, the owner, the original location, the date when it was placed, the name of the employee who placed the label, etc. The necessity of the data owner resides in the need to manage clearly what is preserved/ removed, and if it is found later that it was wrong to sort the object, it can be retrieved and repositioned at the workstation where the target is needed [3].

The concrete stages of this activity are:

1. establishing the person responsible for the sorting activity;

2. setting criteria for the sorting activity;

3. setting the day and time of the event;

4. establishing the temporary holding area (quarantine area) is usually done by consulting the employees of the sector/ structure and in marking this area a black and white adhesive tape is used and labels (panels) are placed on the nearby walls; 
5. announcing the employees that on ........., in the compartment/sector/office there is a sorting action to which they have to participate in;

6. taking a picture of the initial situation (before sorting begins);

7. performing the sorting activity (the actual labelling) and moving unnecessary objects to the temporary holding area (quarantine area);

8. analysis and registration of objects in the temporary holding area (quarantine area).During the application of the labels, for reasons of operative accounting, a specially designated person assigned to the materials management line shall record in a register the name of the object and the place where it was stored. This is also required for another reason. Sometimes, it may be necessary to recover such items from the temporary holding area, as it may happen that sorting does not correctly assess certain criteria of necessity or frequency of use or average consumption requirements on a certain period of time. These notations will help to find and recover those objects more quickly.

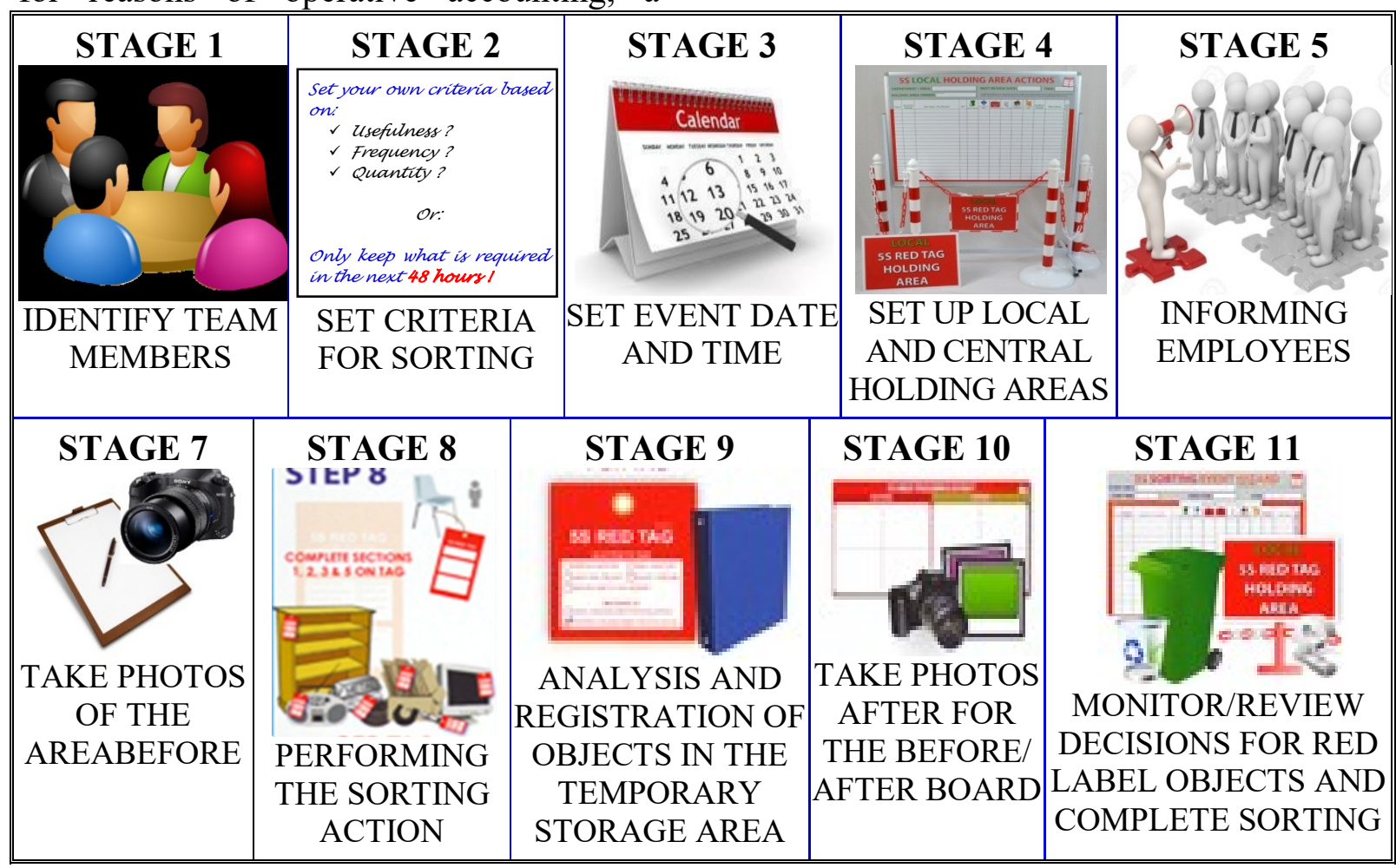

Figure 4 Structure of the sorting stage in the 5S methodology

9. taking photos of the final situation (after completing the actual sorting);

10. monitoring/reviewing decisions for red label objects and completing sorting. For the temporary holding area, a maximum period of time (an average of 2 to 4 weeks) is set in which red-label items can stay in that area. During this period, it is possible to retrieve objects or store others as the actual results of the initial sorting rules are checked in real working conditions. These inward/outward movements of objects are done only by recording in the record register and the $5 \mathrm{~S}$ task manager will periodically check the holding conditions. After the expiration of the storage period, these objects will be removed from the holding area, with action on three potential directions:

10.1. identifying a workstation that needs those objects;

10.2. storage (in the warehouse/archive if lawful prescriptions do not allow them to be destroyed/disposed of by other types of action); 
10.3. removing from the accounting records (return to owners, sale, recycling, donation, repair, scrapping, destruction, etc.).

In conclusion, an important advantage gained through the sorting action is to shorten the time needed to search and to find the necessary objects, with a considerable improvement in the productivity and quality of work, as well as a reduction of the risks of workplace injuries with an effect on the increase of occupational health and safety [2].

\section{Conclusions.}

The first stage, the sorting process, is notonly a removal of unnecessary/waste/ consumed objects/programs, but it also means organization and safety at work done by sustaining and respecting the workplace every day.

This first step is the basis of any $5 \mathrm{~S}$ approach and has as its own objectives:

- better control over the location and position of objects and equipment;

- improving the record of these materials;
- facilitating the implementation of other $5 \mathrm{~S}$ methodology stages in practice.

The benefits of $5 \mathrm{~S}$ can be noticed into increasing the work productivity by:

- increasing the morale of employees as everyone works more efficiently in a job free of unnecessary things;

- reducing inventory levels (by keeping only useful objects in the workplace, inventories can be identified and eliminated);

- eliminating search time for objects/ tools;

- reducing resource waste, wasteful time, physical and mental effort;

- identifying problems, failures or nonconformities fast;

- introducing best practices;

- improving safety and security at work (by creating a visual job, the dangers are easier to identify and prevent);

- increasing the quality of the product/ service and processes because a clean environment and a visual management contribute to preventing mistakes and reducing the waste(scrap).

\section{References}

[1] http://www.kaizen-competitivity.ro/cine_prezinta/prof_masaaki_imai-48-53-ro.html/

[2] http://www.leanblog.ro/wp/instrumente-lean/instrumente-lean/5s/seiri/

[3] C.V. Kifor, C. Oprean, Ingineria calității, Sibiu, "Lucian Blaga" University Publishing House, 2002, pp. 81-82. 